\title{
Stochastic nonautonomous Gompertz model with Lévy jumps
}

\author{
Min Zhu ${ }^{1,2 \dagger}$, Junping $\mathrm{Li}^{1}$ and Xiaoxia Yang ${ }^{1 *+}$
}

"Correspondence:
math921@sina.com
'School of Mathematics and
Statistics, Central South University,
Changsha, 410083, China
Full list of author information is
available at the end of the article
${ }^{+}$Equal contributors

${ }^{\dagger}$ Equal contributors

\begin{abstract}
This paper deals with stochastic nonautonomous Gompertz model with Lévy jumps. To begin with, the existence of a global positive solution and an explicit solution have been derived. In addition, asymptotic moment properties are discussed. Besides, sufficient conditions for extinction, persistence in mean, and weak persistence are obtained. It is proved that the variability of Lévy jumps can affect the asymptotic property of the system.
\end{abstract}

Keywords: Gompertz model; Lévy jumps; persistence; extinction

\section{Introduction}

In mathematical ecology, the Gompertz model is one of the most important models, and it is considered to be one of best fitted to describe growth of certain types of tumor. On the other hand, the Gompertz model is another important type of mathematical models; for example, the growth of the industrial production, the life cycle of a product, and population growth over a certain period all comply with this model [1]. However, environmental noise has very important effects on dynamics of populations in real world. For this reason, in [2], the authors assumed that the growth deceleration factor $b$ did not change while the variability of environmental conditions induced fluctuations in the intrinsic growth rate $a$. Then they proposed a stochastic Gompertz model of the form

$$
\mathrm{d} x(t)=x(t)(a-b \ln x(t)) \mathrm{d} t+\sigma x(t) \mathrm{d} B(t)
$$

where $x(t)$ is the number of cells or population size at time $t, a$ represents the intrinsic growth rate, and $b$ is the growth deceleration factor. Due to the importance both in theory and in applications, the stochastic Gompertz model perturbed by Brownian motion has been studied extensively by many authors, and there is a great amount of literature on this topic; see, for example, [1-6]. Jovanovic and Krstic [4] considered a stochastic Gompertz model with time delay and gave sufficient conditions for the persistence in mean and extinction; $\mathrm{Hu}$ [1] discussed the asymptotic behaviors of a stochastic Gompertz model with Markovian switching. Furthermore, from the viewpoint of biology, large and sudden environmental disturbance, such as earthquakes, tsunamis, hurricanes, floods, or droughts may have important consequences on the system. These phenomena cannot be exactly depicted by Brownian motion. To explain these phenomena, introducing a jump process

(c) 2016 Zhu et al. This article is distributed under the terms of the Creative Commons Attribution 4.0 International License (http://creativecommons.org/licenses/by/4.0/), which permits unrestricted use, distribution, and reproduction in any medium, provided you give appropriate credit to the original author(s) and the source, provide a link to the Creative Commons license, and indicate if changes were made. 
into this system may be one of the most effective methods. There are a large number of literature on this topic, for example, Bao et al. [7-13], Hou-Bao [14], Liu [15, 16], and the references therein. As a result of the mentioned themes, this paper puts forward a stochastic nonautonomous Gompertz model driven by Lévy jumps of the form

$$
\mathrm{d} x(t)=a(t) x(t) \ln \left(\frac{b(t)}{x(t)}\right) \mathrm{d} t+\sigma(t) x(t) \mathrm{d} B(t)+\int_{\mathbb{Y}} x\left(t^{-}\right) h(t, u) \tilde{N}(\mathrm{~d} t, \mathrm{~d} u)
$$

with $x(0)=x_{0}>0$, where $a(\cdot)$ represents the intrinsic growth rate, $b(\cdot)$ is the plateau number of cells or population size, $x\left(t^{-}\right)$is the left limit of $x(t),\{B(t), t \geq 0\}$ is a real-valued standard Brownian motion, and $N$ is a Poisson counting measure with characteristic measure $\lambda$ on a measurable subset $\mathbb{Y}$ of $[0, \infty)$ with $\lambda(\mathbb{Y})<\infty$ and $\tilde{N}(\mathrm{~d} t, \mathrm{~d} u):=N(\mathrm{~d} t, \mathrm{~d} u)-\lambda(\mathrm{d} u) \mathrm{d} t$. Throughout this paper, the process $\{B(t), t \geq 0\}$ is defined on a complete probability space $(\Omega, \mathscr{F}, \mathbb{P})$ with a filtration $\left\{\mathscr{F}_{t}\right\}_{t \geq 0}$ satisfying the usual conditions (i.e., it is right continuous and increasing, and $\mathscr{F}_{0}$ contains all $\mathbb{P}$-null sets) and is independent of $N$. The parameters $a(\cdot)$ and $b(\cdot)$ are positive functions on $\mathbb{R}_{+}$, and the function $h: \mathbb{Y} \times(0, \infty) \rightarrow \mathbb{R}$ is bounded and continuous with respect to $\lambda$ and is $\mathfrak{B}(\mathbb{Y}) \times \mathscr{F}_{t}$-measurable.

Although there have been many excellent works on the stochastic Gompertz model, studies on a stochastic Gompertz model with jumps have not been done yet to the best of our knowledge. Therefore, this study has some practical significance. First, we introduce the following technique result from Bao et al. [9] for the jump-diffusion coefficient.

Assumption A.1 For any $t \geq 0, a(t)>0, b(t)>0, \sigma(t)>0$, and $h(t, u)$ are bounded functions, and $h(t, u)>-1, u \in \mathbb{Y}$.

Assumption A.2 There exist a constant $C>0$ such that

$$
\sup _{t \geq 0}|c(t)| \leq C
$$

where

$$
c(t):=a(t) \ln b(t)-\frac{\sigma^{2}(t)}{2}-\int_{\mathbb{Y}}[h(t, u)-\ln (1+h(t, u))] \lambda(\mathrm{d} u) .
$$

Assumption A.3 There exist a constant $L>0$ such that, for any $t \geq 0$,

$$
\int_{\mathbb{Y}}(\ln (1+h(t, u)))^{2} \lambda(\mathrm{d} u) \leq L
$$

For notational simplicity, we introduce the following symbols:

$$
\hat{\varphi}:=\inf _{t \geq 0} \varphi(t), \quad \check{\varphi}:=\sup _{t \geq 0} \varphi(t) .
$$

\section{Global positive solution}

To get the conclusion about a global positive solution, we need the following useful lemma.

Lemma 2.1 Let Assumption A.1 hold. Then, for all initial values $x_{0} \in \mathbb{R}_{+}$,

$$
\mathbb{P}\{x(t)>0 \text { for } t>0\}=1 .
$$


Proof Denote $\tau:=\inf \{t>0 ; x(t) \leq 0\}$. If (2.1) were false, there would exist some $x_{0}>0$ such that $\mathbb{P}\{\tau<\infty\}>0$. So here is a pair of constants $T>0$ and $K>0$ sufficiently large for $\mathbb{P}(A)>0$, where

$$
A:=\{\tau \leq T, 0<x(t) \leq K \text { for all } 0<t \leq \tau\} .
$$

Now, for any $\varepsilon \in\left(0, x_{0}\right)$, define the stopping time

$$
\tau_{\varepsilon}=\inf \{t>0 ; x(t) \notin(\varepsilon, K+1)\} .
$$

Then for $0<t \leq T, 0<x(t) \leq K+1$, by Itô's formula, for $p>0$ such that $-p \hat{a} \ln \hat{b}+\frac{p(p+1)}{2} \check{\sigma}^{2}+$ $\int_{\mathbb{Y}}\left[(1+\hat{h}(u))^{-p}-1+p \breve{h}(u)\right] \lambda(\mathrm{d} u)>0$, we deduce

$$
\begin{aligned}
x^{-p}\left(T \wedge \tau_{\varepsilon}\right)= & x_{0}^{-p}+\int_{0}^{T \wedge \tau_{\varepsilon}} x^{-p}(s)\left\{p a(s)(\ln x(s)-\ln b(s))+\frac{p(p+1)}{2} \sigma^{2}(s)\right. \\
& \left.+\int_{\mathbb{Y}}\left[(1+h(s, u))^{-p}-1+p h(s, u)\right] \lambda(\mathrm{d} u)\right\} \mathrm{d} s+\int_{0}^{T \wedge \tau_{\varepsilon}}-p x^{-p}(s) \sigma(s) \mathrm{d} B(s) \\
& +\int_{0}^{T \wedge \tau_{\varepsilon}} \int_{\mathbb{Y}} x^{-p}(s)\left[(1+h(s, u))^{-p}-1\right] \tilde{N}(\mathrm{~d} s, \mathrm{~d} u) \\
\leq & x_{0}^{-p}+\int_{0}^{T \wedge \tau_{\varepsilon}} x^{-p}(s)\left\{p \check{a} \ln (K+1)-p \hat{a} \ln \hat{b}+\frac{p(p+1)}{2} \check{\sigma}^{2}\right. \\
& \left.+\int_{\mathbb{Y}}\left[(1+\hat{h}(u))^{-p}-1+p \check{h}(u)\right] \lambda(\mathrm{d} u)\right\} \mathrm{d} s+\int_{0}^{T \wedge \tau_{\varepsilon}}-p x^{-p}(s) \sigma(s) \mathrm{d} B(s) \\
& +\int_{0}^{T \wedge \tau_{\varepsilon}} \int_{\mathbb{Y}} x^{-p}(s)\left[(1+h(s, u))^{-p}-1\right] \tilde{N}(\mathrm{~d} s, \mathrm{~d} u) .
\end{aligned}
$$

Taking the expectation of both sides and applying Gronwall's inequality yield that

$$
\begin{aligned}
\mathbb{E} x^{-p}\left(T \wedge \tau_{\varepsilon}\right) \leq & x_{0}^{-p} \exp \left\{\left[p \check{a} \ln (K+1)-p \hat{a} \ln \hat{b}+\frac{p(p+1)}{2} \check{\sigma}^{2}\right.\right. \\
& \left.\left.+\int_{\mathbb{Y}}\left[(1+\hat{h}(u))^{-p}-1+p \check{h}(u)\right] \lambda(\mathrm{d} u)\right] T\right\} .
\end{aligned}
$$

On the other hand, for any $\omega \in A, \tau_{\varepsilon} \leq T$ and $x\left(\tau_{\varepsilon}\right)=\varepsilon$. Then, for any $\varepsilon \in\left(0, x_{0}\right)$,

$$
\begin{aligned}
0< & \mathbb{P}(A) \leq \varepsilon^{p} \mathbb{E}\left[x^{-p}\left(T \wedge \tau_{\varepsilon}\right) \mathbf{I}_{A}\right] \leq \varepsilon^{p} \mathbb{E}\left[x^{-p}\left(T \wedge \tau_{\varepsilon}\right)\right] \\
\leq & \varepsilon^{p} x_{0}^{-p} \exp \left\{\left[p \check{a} \ln (K+1)-p \hat{a} \ln \hat{b}+\frac{p(p+1)}{2} \check{\sigma}^{2}\right.\right. \\
& \left.\left.+\int_{\mathbb{Y}}\left[(1+\hat{h}(u))^{-p}-1+p \check{h}(u)\right] \lambda(\mathrm{d} u)\right] T\right\} .
\end{aligned}
$$

Letting $\varepsilon \downarrow 0$ yields $\mathbb{P}(A)=0$. We get a contradiction. The proof is complete.

Remark 2.1 Since $x(t)$ in (1.1) is the number of cells or population size at time $t$, we are only interested in the positive solutions. Lemma 2.1 ensures this point from the angle of theory, and it reveals that almost all sample paths of any solution starting from a positive state will never be nonpositive. 
Theorem 2.1 Under Assumptions A.1 and A.2, for any initial value $x_{0} \in \mathbb{R}_{+}$, there is a unique global solution $x(t) \in \mathbb{R}_{+}$on $t \geq 0$ almost surely. The unique positive solution can be expressed as

$$
\begin{aligned}
\ln x(t)= & \exp \left(-\int_{0}^{t} a(s) \mathrm{d} s\right)\left[\ln x_{0}+\int_{0}^{t} \exp \left(\int_{0}^{s} a(r) \mathrm{d} r\right) c(s) \mathrm{d} s\right. \\
& +\int_{0}^{t} \exp \left(\int_{0}^{s} a(r) \mathrm{d} r\right) \sigma(s) \mathrm{d} B(s) \\
& \left.+\int_{0}^{t} \exp \left(\int_{0}^{s} a(r) \mathrm{d} r\right) \int_{\mathbb{Y}} \ln (1+h(s, u)) \tilde{N}(\mathrm{~d} s, \mathrm{~d} u)\right] .
\end{aligned}
$$

Proof We divide the proof into two steps.

Step 1. We first claim that there exists a unique global solution $x(t) \in \mathbb{R}_{+}$by means of the transform $X(t)=\ln x(t)$. By Lemma 2.1 this transform makes sense for all $t \geq 0$ almost everywhere. Then $X(t)$ satisfies the following equation:

$$
\mathrm{d} X(t)=[c(t)-a(t) X(t)] \mathrm{d} t+\sigma(t) \mathrm{d} B(t)+\int_{\mathbb{Y}} \ln (1+h(t, u)) \tilde{N}(\mathrm{~d} t, \mathrm{~d} u)
$$

on $t \geq 0$ with initial value $X(0)=\ln x_{0}$, where $c(t)$ is introduced in Assumption A.2. It is easy to find that if the coefficients of (2.3) satisfy the global Lipschitz condition and linear growth condition, then for any initial value $X(0) \in \mathbb{R}_{+}$, there is a unique global solution $X(t)$ on $t \geq 0$. Therefore, by Itô's formula, $x(t)=\mathrm{e}^{X(t)}$ is a unique positive global solution of (1.1) with initial value $x_{0} \in \mathbb{R}_{+}$.

Step 2. We first investigate the explicit solution of Eq. (2.3), and by making the change of variable, we will get that Eq. (1.1) has an explicit solution, which can be expressed as Eq. (2.2). By the variation-of-constants formula ([9], Lemma 4.1) Eq. (2.3) has a unique solution $X(t), t \geq 0$, which can be expressed as

$$
\begin{aligned}
X(t)= & \exp \left(-\int_{0}^{t} a(s) \mathrm{d} s\right)\left[X(0)+\int_{0}^{t} \exp \left(\int_{0}^{s} a(r) \mathrm{d} r\right) c(s) \mathrm{d} s\right. \\
& +\int_{0}^{t} \exp \left(\int_{0}^{s} a(r) \mathrm{d} r\right) \sigma(s) \mathrm{d} B(s) \\
& \left.+\int_{0}^{t} \exp \left(\int_{0}^{s} a(r) \mathrm{d} r\right) \int_{\mathbb{Y}} \ln (1+h(s, u)) \tilde{N}(\mathrm{~d} s, \mathrm{~d} u)\right]
\end{aligned}
$$

From this it follows immediately that, for any $t \geq 0$,

$$
\begin{aligned}
\ln x(t)= & \exp \left(-\int_{0}^{t} a(s) \mathrm{d} s\right)\left[\ln x_{0}+\int_{0}^{t} \exp \left(\int_{0}^{s} a(r) \mathrm{d} r\right) c(s) \mathrm{d} s\right. \\
& +\int_{0}^{t} \exp \left(\int_{0}^{s} a(r) \mathrm{d} r\right) \sigma(s) \mathrm{d} B(s) \\
& \left.+\int_{0}^{t} \exp \left(\int_{0}^{s} a(r) \mathrm{d} r\right) \int_{\mathbb{Y}} \ln (1+h(s, u)) \tilde{N}(\mathrm{~d} s, \mathrm{~d} u)\right] .
\end{aligned}
$$

Next, we show that the positive solution satisfies the following result if the jumpdiffusion coefficient is controlled under a certain range. 
Theorem 2.2 Let Assumptions A.1 and A.2 hold, and assume further that $\mathrm{e}^{-1}-1<h(t, u)<$ $\mathrm{e}-1$ for any initial value $x_{0} \in \mathbb{R}_{+}$.Then the solution $x(t) \in \mathbb{R}_{+}$to Eq. (1.1) satisfies

$$
\Phi^{-1}(t)\left(\frac{1}{x_{0}}+\int_{0}^{t} \Phi^{-1}(s) a(s) \mathrm{d} s\right)^{-1} \leq x(t) \leq \Psi(t)\left(x_{0}+\int_{0}^{t} \Psi^{-1}(s) a(s) b(s) \mathrm{d} s\right),
$$

where

$$
\begin{aligned}
\Phi(t):= & \exp \left\{\int _ { 0 } ^ { t } \left[-a(t)(1+\ln b(t))+\frac{\sigma^{2}(t)}{2}\right.\right. \\
& \left.+\int_{\mathbb{Y}}(h(s, u)+\ln (1-\ln (1+h(s, u)))) \lambda(\mathrm{d} u)\right] \mathrm{d} s \\
& \left.-\int_{0}^{t} \sigma(s) \mathrm{d} B(s)+\int_{0}^{t} \int_{\mathbb{Y}} \ln (1-\ln (1+h(s, u))) \tilde{N}(\mathrm{~d} s, \mathrm{~d} u)\right\}
\end{aligned}
$$

and

$$
\begin{aligned}
\Psi(t):= & \exp \left\{-\int_{0}^{t}\left[a(t)+\sigma^{2}(t)+\int_{\mathbb{Y}}(h(s, u)-\ln (1+\ln (1+h(s, u)))) \lambda(\mathrm{d} u)\right] \mathrm{d} s\right. \\
& \left.+\int_{0}^{t} \sigma(s) \mathrm{d} B(s)+\int_{0}^{t} \int_{\mathbb{Y}} \ln (1+\ln (1+h(s, u))) \tilde{N}(\mathrm{~d} s, \mathrm{~d} u)\right\} .
\end{aligned}
$$

Proof Using Itô's formula, we have

$$
\begin{aligned}
\ln x(t)= & \ln x_{0}+\int_{0}^{t}\left[a(s) \ln \frac{b(s)}{x(s)}-\frac{\sigma^{2}(s)}{2}-\int_{\mathbb{Y}}(h(s, u)-\ln (1+h(s, u))) \lambda(\mathrm{d} u)\right] \mathrm{d} s \\
& +\int_{0}^{t} \sigma(s) \mathrm{d} B(s)+\int_{0}^{t} \int_{\mathbb{Y}} \ln (1+h(s, u)) \tilde{N}(\mathrm{~d} s, \mathrm{~d} u) \\
\leq & \ln x_{0}+\int_{0}^{t}\left[a(s) \frac{b(s)}{x(s)}-a(s)-\frac{\sigma^{2}(s)}{2}\right. \\
& \left.-\int_{\mathbb{Y}}(h(s, u)-\ln (1+h(s, u))) \lambda(\mathrm{d} u)\right] \mathrm{d} s+\int_{0}^{t} \sigma(s) \mathrm{d} B(s) \\
& +\int_{0}^{t} \int_{\mathbb{Y}} \ln (1+h(s, u)) \widetilde{N}(\mathrm{~d} s, \mathrm{~d} u) .
\end{aligned}
$$

Here we have used the inequality $\ln (x+1) \leq x, x>0$. Now consider the following auxiliary process with jumps:

$$
\begin{aligned}
\ln y(t)= & \ln y(0)+\int_{0}^{t}\left[a(s) \frac{b(s)}{y(s)}-a(s)-\frac{\sigma^{2}(s)}{2}-\int_{\mathbb{Y}}(h(s, u)\right. \\
& -\ln (1+h(s, u))) \lambda(\mathrm{d} u)] \mathrm{d} s+\int_{0}^{t} \sigma(s) \mathrm{d} B(s) \\
& +\int_{0}^{t} \int_{\mathbb{Y}} \ln (1+h(s, u)) \tilde{N}(\mathrm{~d} s, \mathrm{~d} u), \\
\ln y(0)= & \ln x_{0} .
\end{aligned}
$$

Then by comparison theorem [17], $\ln x(t) \leq \ln y(t)$ a.s. for all $t \geq 0$. In what follows, we shall get an explicit solution of Eq. (2.4). Making change of variable $z(t)=\ln y(t)$ and multiplying 
both sides by $\mathrm{e}^{z(t)}$ yield that

$$
\begin{aligned}
\mathrm{de}^{z(t)}= & {\left[a(t) b(t)-\mathrm{e}^{z(t)}\left(a(t)+\frac{\sigma^{2}(t)}{2}+\int_{\mathbb{Y}}(h(t, u)-\ln (1+h(t, u))) \lambda(\mathrm{d} u)\right)\right] \mathrm{d} t } \\
& +\mathrm{e}^{z(t)} \sigma(t) \mathrm{d} B(t)+\int_{\mathbb{Y}} \mathrm{e}^{z(t)} \ln (1+h(t, u)) \tilde{N}(\mathrm{~d} t, \mathrm{~d} u) .
\end{aligned}
$$

Using the same method to the above equation yields that

$$
\begin{aligned}
\mathrm{d} X(t)= & {\left[a(t) b(t)-X(t)\left(a(t)+\frac{\sigma^{2}(t)}{2}+\int_{\mathbb{Y}}(h(t, u)-\ln (1+h(t, u))) \lambda(\mathrm{d} u)\right)\right] \mathrm{d} t } \\
& +X(t) \sigma(t) \mathrm{d} B(t)+\int_{\mathbb{Y}} X(t) \ln (1+h(t, u)) \tilde{N}(\mathrm{~d} t, \mathrm{~d} u),
\end{aligned}
$$

which has a solution of the form

$$
X(t)=\Psi(t)\left(X(0)+\int_{0}^{t} \Psi^{-1}(s) a(s) b(s) \mathrm{d} s\right),
$$

where

$$
\begin{aligned}
\Psi(t):= & \exp \left\{-\int_{0}^{t}\left[a(t)+\sigma^{2}(t)+\int_{\mathbb{Y}}(h(s, u)-\ln (1+\ln (1+h(s, u)))) \lambda(\mathrm{d} u)\right] \mathrm{d} s\right. \\
& \left.+\int_{0}^{t} \sigma(s) \mathrm{d} B(s)+\int_{0}^{t} \int_{\mathbb{Y}} \ln (1+\ln (1+h(s, u))) \tilde{N}(\mathrm{~d} s, \mathrm{~d} u)\right\} .
\end{aligned}
$$

Consequently, from the above arguments we get $x(t) \leq y(t)$ and $y(t)=X(t), t \geq 0$, a.s. On the other hand,

$$
\begin{aligned}
\ln x(t) \geq & \ln x_{0}+\int_{0}^{t}\left[a(s)(\ln b(s)+1)-a(s) x(s)-\frac{\sigma^{2}(s)}{2}\right. \\
& \left.-\int_{\mathbb{Y}}(h(s, u)-\ln (1+h(s, u))) \lambda(\mathrm{d} u)\right] \mathrm{d} s+\int_{0}^{t} \sigma(s) \mathrm{d} B(s) \\
& +\int_{0}^{t} \int_{\mathbb{Y}} \ln (1+h(s, u)) \tilde{N}(\mathrm{~d} s, \mathrm{~d} u) .
\end{aligned}
$$

Using the same arguments as those used to obtain $x(t) \leq y(t)$, we have

$$
x(t) \geq \Phi^{-1}(t)\left(\frac{1}{x_{0}}+\int_{0}^{t} \Phi^{-1}(s) a(s) \mathrm{d} s\right)^{-1},
$$

where

$$
\begin{aligned}
\Phi(t):= & \exp \left\{\int _ { 0 } ^ { t } \left[-a(t)(1+\ln b(t))+\frac{\sigma^{2}(t)}{2}\right.\right. \\
& \left.+\int_{\mathbb{Y}}(h(s, u)+\ln (1-\ln (1+h(s, u)))) \lambda(\mathrm{d} u)\right] \mathrm{d} s \\
& \left.-\int_{0}^{t} \sigma(s) \mathrm{d} B(s)+\int_{0}^{t} \int_{\mathbb{Y}} \ln (1-\ln (1+h(s, u))) \tilde{N}(\mathrm{~d} s, \mathrm{~d} u)\right\} .
\end{aligned}
$$


Remark 2.2 Theorem 2.2 shows that a Lévy noise can suppress a potential explosion, but with weaker conditions imposed on the diffusion coefficient $\sigma(\cdot)$. Under Assumption A.1, when $-1<h(\cdot)<0$, the disturbance denotes decreasing of the community, whereas when $h(\cdot)>0$, it respects increasing.

\section{Asymptotic moment properties}

From the previous result we know that for any initial value, Eq. (1.1) has a unique solution, which can be expressed as Eq. (2.2). In the following, by its explicit solution we first aim to find the $p$ th moment of the solution and discuss the long-term behavior.

Theorem 3.1 Let the conditions of Theorem 2.1 hold. Assume further that, for any $p>0$,

$$
\begin{aligned}
& \sup _{t \geq 0} \int_{0}^{t} \int_{\mathbb{Y}}\left[(1+h(s, u))^{p \exp \left(-\int_{s}^{t} a(r) \mathrm{d} r\right)}-1\right. \\
& \left.\quad-p \exp \left(-\int_{s}^{t} a(r) \mathrm{d} r\right) \ln (1+h(s, u))\right] \lambda(\mathrm{d} u) \mathrm{d} s<\infty .
\end{aligned}
$$

Then there is a constant $K(p)>0$ such that

$$
\limsup _{t \rightarrow \infty} E x^{p}(t) \leq K(p)<\infty
$$

Proof Noting that Eq. (2.2) is an explicit solution to Eq. (1.1), we derive that

$$
\begin{aligned}
\ln x^{p}(t)= & p \exp \left(-\int_{0}^{t} a(s) \mathrm{d} s\right) \ln x_{0}+p \int_{0}^{t} \exp \left(-\int_{s}^{t} a(r) \mathrm{d} r\right) c(s) \mathrm{d} s \\
& +p \int_{0}^{t} \exp \left(-\int_{s}^{t} a(r) \mathrm{d} r\right) \sigma(s) \mathrm{d} B(s) \\
& +p \int_{0}^{t} \int_{\mathbb{Y}} \exp \left(-\int_{s}^{t} a(r) \mathrm{d} r\right) \ln (1+h(s, u)) \tilde{N}(\mathrm{~d} s, \mathrm{~d} u) \\
\leq & p \exp \left(-\int_{0}^{t} a(s) \mathrm{d} s\right) \ln x_{0}+p \int_{0}^{t} \exp \left(-\int_{s}^{t} a(r) \mathrm{d} r\right) c(s) \mathrm{d} s \\
& +p \int_{0}^{t} \mathrm{e}^{\hat{a}(s-t)} \check{\sigma} \mathrm{d} B(s) \\
& +p \int_{0}^{t} \int_{\mathbb{Y}} \exp \left(-\int_{s}^{t} a(r) \mathrm{d} r\right) \ln (1+h(s, u)) \tilde{N}(\mathrm{~d} s, \mathrm{~d} u),
\end{aligned}
$$

which means that

$$
\begin{aligned}
x^{p}(t) \leq & \exp \left\{p \exp \left(-\int_{0}^{t} a(s) \mathrm{d} s\right) \ln x_{0}\right\} \exp \left\{\int_{0}^{t} p \mathrm{e}^{\hat{a}(s-t)} \check{\sigma} \mathrm{d} B(s)\right\} \\
& \times \exp \left\{p \int_{0}^{t} \exp \left(-\int_{s}^{t} a(r) \mathrm{d} r\right) c(s) \mathrm{d} s\right\} \\
& \times \exp \left\{p \int_{0}^{t} \int_{\mathbb{Y}} \exp \left(-\int_{s}^{t} a(r) \mathrm{d} r\right) \ln (1+h(s, u)) \tilde{N}(\mathrm{~d} s, \mathrm{~d} u)\right\} .
\end{aligned}
$$


Let $M_{1}(t)=\int_{0}^{t} p \mathrm{e}^{\hat{a}(s-t)} \check{\sigma} \mathrm{d} B(s)$. Then $M_{1}(t)$ is a real-valued continuous martingale, and the quadratic variation of $M_{1}(t)$ is

$$
\left[M_{1}\right](t)=\int_{0}^{t} p^{2} \check{\sigma}^{2} \mathrm{e}^{2 \hat{a}(s-t)} \mathrm{d} s=\frac{p^{2} \check{\sigma}^{2}}{2 \hat{a}}\left(1-\mathrm{e}^{-2 \hat{a} t}\right) .
$$

Taking expectations on both sides of Eq. (3.2), we deduce

$$
\begin{aligned}
E x^{p}(t) \leq & \exp \left\{p \exp \left(-\int_{0}^{t} a(s) \mathrm{d} s\right) \ln x_{0}\right\} \exp \left\{\frac{p^{2} \check{\sigma}^{2}}{4 \hat{a}}\left(1-\mathrm{e}^{-2 \hat{a} t}\right)\right\} \\
& \times \exp \left\{p \int_{0}^{t} \exp \left(-\int_{s}^{t} a(r) \mathrm{d} r\right) c(s) \mathrm{d} s\right\} \\
& \times \exp \left\{\int _ { 0 } ^ { t } \int _ { \mathbb { Y } } \left[(1+h(s, u))^{p \exp \left(-\int_{s}^{t} a(r) \mathrm{d} r\right)}-1\right.\right. \\
& \left.\left.-p \exp \left(-\int_{s}^{t} a(r) \mathrm{d} r\right) \ln (1+h(s, u))\right] \lambda(\mathrm{d} u) \mathrm{d} s\right\} .
\end{aligned}
$$

Here we have used the fact that, by the Gardiner relation,

$$
\begin{aligned}
E\left[\mathrm{e}^{M_{1}(t)}\right] & =\exp \left\{\frac{1}{2} E\left[M_{1}(t)\right]^{2}\right\}=\exp \left\{\frac{1}{2} \int_{0}^{t} p^{2} \check{\sigma}^{2} \mathrm{e}^{2 \hat{a}(s-t)} \mathrm{d} s\right\} \\
& =\exp \left\{\frac{p^{2} \check{\sigma}^{2}}{4 \hat{a}}\left(1-\mathrm{e}^{-2 \hat{a} t}\right)\right\}
\end{aligned}
$$

and that, by Theorem 2.3.7(1) in [18],

$$
\begin{gathered}
E\left[\exp \left\{p \int_{0}^{t} \int_{\mathbb{Y}} \exp \left(-\int_{s}^{t} a(r) \mathrm{d} r\right) \ln (1+h(s, u)) \tilde{N}(\mathrm{~d} s, \mathrm{~d} u)\right\}\right] \\
=\exp \left\{\int _ { 0 } ^ { t } \int _ { \mathbb { Y } } \left[(1+h(s, u))^{p \exp \left(-\int_{s}^{t} a(r) \mathrm{d} r\right)}-1\right.\right. \\
\left.\left.\quad-p \exp \left(-\int_{s}^{t} a(r) \mathrm{d} r\right) \ln (1+h(s, u))\right] \lambda(\mathrm{d} u) \mathrm{d} s\right\} .
\end{gathered}
$$

By Assumptions A.1 and A.2 and by (3.1) we can deduce that there exists a constant $K(p)>$ 0 such that

$$
\limsup _{t \rightarrow \infty} E x^{p}(t) \leq K(p)<\infty
$$

Remark 3.1 Under a suitable condition, we have shown that, for any $p>0$, the $p$ th moment of the solution $x(t)$ to Eq. (1.1) is bounded. The conclusion reveals the important fact that a Brownian motion noise and a Lévy noise can suppress a potential explosion.

Remark 3.2 If $a, b, \sigma, h$ are time independent, then Eq. (1.1) reduces to

$$
\mathrm{d} x(t)=a x(t) \ln \left(\frac{b}{x(t)}\right) \mathrm{d} t+\sigma x(t) \mathrm{d} B(t)+\int_{\mathbb{Y}} x\left(t^{-}\right) h(u) \tilde{N}(\mathrm{~d} t, \mathrm{~d} u)
$$


with initial value $x_{0}>0$. It is obvious that the solution of Eq. (3.3) is a time-homogeneous Itô diffusion process. In view of the proof of Theorem 3.1, we can get the population mean. Making use of the expression of the solution of Eq. (1.1) in Theorem 2.1, we show that the solution of Eq. (3.5) can be explicitly expressed by

$$
\begin{aligned}
x(t)= & \exp \left\{\mathrm{e}^{-a t} \ln x_{0}+\frac{c}{a}\left(1-\mathrm{e}^{-a t}\right)+\int_{0}^{t} \mathrm{e}^{a(s-t)} \sigma \mathrm{d} B(s)\right. \\
& \left.+\int_{0}^{t} \int_{\mathbb{Y}} \mathrm{e}^{a(s-t)} \ln (1+h(u)) \tilde{N}(\mathrm{~d} t, \mathrm{~d} u)\right\},
\end{aligned}
$$

where $c:=a \ln b-\frac{\sigma^{2}}{2}-\int_{\mathbb{Y}}[h(u)-\ln (1+h(u))] \lambda(\mathrm{d} u)$.

By the same argument as in Theorem 3.1 we have

$$
\begin{aligned}
E x(t)= & x_{0}^{\mathrm{e}^{-a t}} \exp \left\{\frac{a \ln b-0.5 \sigma^{2}-\int_{\mathbb{Y}}[h(u)-\ln (1+h(u))] \lambda(\mathrm{d} u)}{a}\left(1-\mathrm{e}^{-a t}\right)\right. \\
& \left.+\frac{\sigma^{2}}{4 a}\left(1-\mathrm{e}^{-2 a t}\right)+\int_{0}^{t} \int_{\mathbb{Y}}\left[(1+h(u))^{\mathrm{e}^{a(s-t)}}-1-\mathrm{e}^{a(s-t)} \ln (1+h(u))\right] \lambda(\mathrm{d} u) \mathrm{d} s\right\} .
\end{aligned}
$$

Therefore, we can control the mean value of the population in view of the representation of the above equality, for example, we can increase the number $b$ to increase the mean value of the population under fixed Brownian motion noise and Lévy noise.

Another important property of the solution is that it is $p$ th moment exponentially stable. To be precise, let us give the definition.

Definition 3.1 The solution of (1.1) is said to be $p$ th moment exponentially stable if

$$
\limsup _{t \rightarrow \infty} \frac{1}{t} \ln \left(E|x(t)|^{p}\right)<0
$$

for all $x_{0} \in \mathbb{R}_{+}$.

Theorem 3.2 Let the conditions of Theorem 3.1 hold. The pth moment of the solution $x(t)$ has the property

$$
\limsup _{t \rightarrow \infty} \frac{1}{t} \ln \left(E|x(t)|^{p}\right) \leq \limsup _{t \rightarrow \infty} \frac{p}{t} \int_{0}^{t} \exp \left(-\int_{s}^{t} a(r) \mathrm{d} r\right) c(s) \mathrm{d} s
$$

Therefore, the solution of (1.1) is pth moment exponentially stable if and only if

$$
\limsup _{t \rightarrow \infty} \frac{1}{t} \int_{0}^{t} \exp \left(-\int_{s}^{t} a(r) \mathrm{d} r\right) c(s) \mathrm{d} s<0
$$

where, for any $t \geq 0$,

$$
c(t):=a(t) \ln b(t)-\frac{\sigma^{2}(t)}{2}-\int_{\mathbb{Y}}[h(t, u)-\ln (1+h(t, u))] \lambda(\mathrm{d} u) .
$$


Proof Observe that (3.3) can be rewritten in the form

$$
\begin{aligned}
\ln E x^{p}(t) \leq & p \exp \left(-\int_{0}^{t} a(s) \mathrm{d} s\right) \ln x_{0}+p \int_{0}^{t} \exp \left(-\int_{s}^{t} a(r) \mathrm{d} r\right) c(s) \mathrm{d} s \\
& +\frac{p^{2} \check{\sigma}^{2}}{4 \hat{a}}\left(1-\mathrm{e}^{-2 \hat{a} t}\right)+\int_{0}^{t} \int_{\mathbb{Y}}\left[(1+h(s, u))^{p \exp \left(-\int_{s}^{t} a(r) \mathrm{d} r\right)}-1\right. \\
& \left.-p \exp \left(-\int_{s}^{t} a(r) \mathrm{d} r\right) \ln (1+h(s, u))\right] \lambda(\mathrm{d} u) \mathrm{d} s
\end{aligned}
$$

and further that

$$
\begin{aligned}
\frac{1}{t} \ln E x^{p}(t) \leq & \frac{p}{t} \exp \left(-\int_{0}^{t} a(s) \mathrm{d} s\right) \ln x_{0}+\frac{p}{t} \int_{0}^{t} \exp \left(-\int_{s}^{t} a(r) \mathrm{d} r\right) c(s) \mathrm{d} s \\
& +\frac{p}{t} \frac{p^{2} \check{\sigma}^{2}}{4 \hat{a}}\left(1-\mathrm{e}^{-2 \hat{a} t}\right) \\
& +\frac{p}{t} \int_{0}^{t} \int_{\mathbb{Y}}\left[(1+h(s, u))^{p \exp \left(-\int_{s}^{t} a(r) \mathrm{d} r\right)}-1\right. \\
& \left.-p \exp \left(-\int_{s}^{t} a(r) \mathrm{d} r\right) \ln (1+h(s, u))\right] \lambda(\mathrm{d} u) \mathrm{d} s .
\end{aligned}
$$

Combining this with Assumption A.1 and (3.1), we then get

$$
\limsup _{t \rightarrow \infty} \frac{1}{t} \ln E x^{p}(t) \leq \limsup _{t \rightarrow \infty} \frac{p}{t} \int_{0}^{t} \exp \left(-\int_{s}^{t} a(r) \mathrm{d} r\right) c(s) \mathrm{d} s .
$$

The desired result then follows.

Theorem 3.3 Let the conditions of Theorem 2.1 hold. Then, for any initial value $x_{1}, x_{2} \in \mathbb{R}_{+}$ and compact subset $\mathbb{K}$ of $\mathbb{R}_{+}$, the solution $x(t)$ of Eq. (1.1) has the property

$$
\lim _{t \rightarrow \infty} E\left|x\left(t, x_{1}\right)-x\left(t, x_{2}\right)\right|=0 \quad \text { uniformly in }\left(x_{1}, x_{2}\right) \in \mathbb{K} \times \mathbb{K} .
$$

Proof By (2.2) we derive that

$$
\left|\ln x\left(t, x_{1}\right)-\ln x\left(t, x_{2}\right)\right|=\exp \left(-\int_{0}^{t} a(s) \mathrm{d} s\right)\left|\ln x_{1}-\ln x_{2}\right| .
$$

Since $a(\cdot)$ is bounded, we get

$$
\mathrm{e}^{-\check{a} t}\left|\ln x_{1}-\ln x_{2}\right| \leq\left|\ln x\left(t, x_{1}\right)-\ln x\left(t, x_{2}\right)\right| \leq \mathrm{e}^{-\hat{a} t}\left|\ln x_{1}-\ln x_{2}\right| .
$$

This implies that

$$
\lim _{t \rightarrow \infty}\left|\ln x\left(t, x_{1}\right)-\ln x\left(t, x_{2}\right)\right|=0 .
$$

Therefore, for any compact subset $\mathbb{K}$ of $\mathbb{R}_{+}$,

$$
\lim _{t \rightarrow \infty} E\left|x\left(t, x_{1}\right)-x\left(t, x_{2}\right)\right|=0 \quad \text { uniformly in }\left(x_{1}, x_{2}\right) \in \mathbb{K} \times \mathbb{K}
$$


Remark 3.3 Theorem 3.3 indicates that any two solutions with different initial values attract each other in the sense of mean.

\section{Extinction and persistence}

Extinction and persistence are important properties in population dynamics, which mean that every species will survive or not. In what follows, we will discuss the extinction and persistence of system (1.1).

Theorem 4.1 Let the conditions of Theorem 2.1 hold. Assume further that, for any $t \geq 0$,

$$
\sup _{t \geq 0} \int_{0}^{t} \int_{\mathbb{Y}} \exp \left\{-\int_{s}^{t} a(r) \mathrm{d} r\right\}[h(s, u)-\ln (1+h(s, u))] \lambda(\mathrm{d} u) \mathrm{d} s<\infty
$$

Then, for any initial value $x_{0} \in \mathbb{R}_{+}$, the solution $x(t)$ of (1.1) has the property

$$
\limsup _{t \rightarrow \infty} \frac{\ln x(t)}{\ln t} \leq 1 \quad \text { a.s. }
$$

and therefore

$$
\limsup _{t \rightarrow \infty} \frac{\ln x(t)}{t} \leq 0 \quad \text { a.s. }
$$

Proof From the explicit solution (2.2) of Eq. (1.1) we get that

$$
\begin{aligned}
& \exp \left(\int_{0}^{t} a(s) \mathrm{d} s\right) \ln x(t) \\
& =\ln x_{0}+\int_{0}^{t} \exp \left(\int_{0}^{s} a(r) \mathrm{d} r\right) c(s) \mathrm{d} s+\int_{0}^{t} \exp \left(\int_{0}^{s} a(r) \mathrm{d} r\right) \sigma(s) \mathrm{d} B(s) \\
& \quad+\int_{0}^{t} \exp \left(\int_{0}^{s} a(r) \mathrm{d} r\right) \int_{\mathbb{Y}} \ln (1+h(s, u)) \tilde{N}(\mathrm{~d} s, \mathrm{~d} u) \\
& \leq \ln x_{0}+\int_{0}^{t} \exp \left(\int_{0}^{s} a(r) \mathrm{d} r\right)\left(a(s) b(s)-1-\frac{\sigma^{2}(s)}{2}\right) \mathrm{d} s \\
& \quad+\int_{0}^{t} \exp \left(\int_{0}^{s} a(r) \mathrm{d} r\right) \sigma(s) \mathrm{d} B(s) \\
& \quad+\int_{0}^{t} \exp \left(\int_{0}^{s} a(r) \mathrm{d} r\right) \int_{\mathbb{Y}} \ln (1+h(s, u)) \tilde{N}(\mathrm{~d} s, \mathrm{~d} u)
\end{aligned}
$$

Here, in the last step, we have utilized the inequality $\ln x \leq x-1(x>0)$. Using the exponential martingale inequality with jumps (i.e., Lemma 4.3 in [9]), we get that, for any positive numbers $\alpha, \beta, T$,

$$
\begin{aligned}
& P\left\{\operatorname { s u p } _ { 0 \leq t \leq T } \left[\int_{0}^{t} \exp \left(\int_{0}^{s} a(r) \mathrm{d} r\right) \sigma(s) \mathrm{d} B(s)\right.\right. \\
& \quad-\frac{\alpha}{2} \int_{0}^{t} \exp \left(\int_{0}^{s} 2 a(r) \mathrm{d} r\right) \sigma^{2}(s) \mathrm{d} s \\
& \quad+\int_{0}^{t} \int_{\mathbb{Y}} \exp \left(\int_{0}^{s} a(r) \mathrm{d} r\right) \ln (1+h(s, u)) \tilde{N}(\mathrm{~d} s, \mathrm{~d} u)
\end{aligned}
$$




$$
\begin{aligned}
& -\frac{1}{\alpha} \int_{0}^{t} \int_{\mathbb{Y}}\left[\exp \left\{\alpha \exp \left(\int_{0}^{s} a(r) \mathrm{d} r\right) \ln (1+h(s, u))\right\}\right. \\
& \left.\left.\left.-1-\alpha \exp \left(\int_{0}^{s} a(r) \mathrm{d} r\right) \ln (1+h(s, u))\right] \lambda(\mathrm{d} u) \mathrm{d} s\right] \geq \beta\right\} \leq \mathrm{e}^{-\alpha \beta} .
\end{aligned}
$$

Choose $T=n \gamma, \alpha=\epsilon \exp \left(-\int_{0}^{n \gamma} a(s) \mathrm{d} s\right)$, and $\beta=\frac{\theta \exp \left(\int_{0}^{n \gamma} a(s) \mathrm{d} s\right) \ln n}{\epsilon}$, where $n \in \mathbb{N}, 0<\epsilon<1$, $\gamma>0$, and $\theta>1$. An application of the Borel-Cantelli lemma then yields that there exists $\Omega_{i} \subseteq \Omega$ with $P\left(\Omega_{i}\right)=1$ such that for any $\omega \in \Omega_{i}$, there is an integer $n_{0}=n_{0}(\omega)$ such that

$$
\begin{gathered}
\int_{0}^{t} \exp \left(\int_{0}^{s} a(r) \mathrm{d} r\right) \sigma(s) \mathrm{d} B(s)+\int_{0}^{t} \int_{\mathbb{Y}} \exp \left(\int_{0}^{s} a(r) \mathrm{d} r\right) \ln (1+h(s, u)) \tilde{N}(\mathrm{~d} s, \mathrm{~d} u) \\
\leq \frac{\epsilon \exp \left(-\int_{0}^{n \gamma} a(s) \mathrm{d} s\right)}{2} \int_{0}^{t} \exp \left(\int_{0}^{s} 2 a(r) \mathrm{d} r\right) \sigma^{2}(s) \mathrm{d} s+\frac{1}{\epsilon \exp \left(-\int_{0}^{n \gamma} a(s) \mathrm{d} s\right)} \\
\quad \times \int_{0}^{t} \int_{\mathbb{Y}}\left[(1+h(s, u))^{\epsilon \exp \left(-\int_{0}^{n \gamma} a(s) \mathrm{d} s\right) \exp \left(\int_{0}^{s} a(r) \mathrm{d} r\right)}-1-\epsilon \exp \left(-\int_{0}^{n \gamma} a(s) \mathrm{d} s\right)\right. \\
\left.\quad \times \exp \left(\int_{0}^{s} a(r) \mathrm{d} r\right) \ln (1+h(s, u))\right] \lambda(\mathrm{d} u) \mathrm{d} s+\frac{\theta \exp \left(\int_{0}^{n \gamma} a(s) \mathrm{d} s\right) \ln n}{\epsilon},
\end{gathered}
$$

where $n>n_{0}, 0 \leq t \leq n \gamma$. Furthermore, by the Young inequality we get

$$
\begin{aligned}
\frac{1}{\epsilon \exp \left(-\int_{0}^{n \gamma} a(s) \mathrm{d} s\right) \exp \left(\int_{0}^{t} a(s) \mathrm{d} s\right)} \\
\quad \times \int_{0}^{t} \int_{\mathbb{Y}}\left[(1+h(s, u)) \exp \left(-\int_{0}^{n \gamma} a(s) \mathrm{d} s\right) \exp \left(\int_{0}^{s} a(r) \mathrm{d} r\right)-1\right. \\
\left.\quad-\epsilon \exp \left(-\int_{0}^{n \gamma} a(s) \mathrm{d} s\right) \exp \left(\int_{0}^{s} a(r) \mathrm{d} r\right) \ln (1+h(s, u))\right] \lambda(\mathrm{d} u) \mathrm{d} s \\
\leq \frac{1}{\epsilon \exp \left(-\int_{0}^{n \gamma} a(s) \mathrm{d} s\right) \exp \left(\int_{0}^{t} a(s) \mathrm{d} s\right)} \int_{0}^{t} \int_{\mathbb{Y}}\left[\epsilon \exp \left(-\int_{0}^{n \gamma} a(s) \mathrm{d} s\right)\right. \\
\quad \times \exp \left(\int_{0}^{s} a(r) \mathrm{d} r\right)[h(s, u)-\ln (1+h(s, u))] \lambda(\mathrm{d} u) \mathrm{d} s \\
=\int_{0}^{t} \int_{\mathbb{Y}} \exp \left(-\int_{s}^{t} a(r) \mathrm{d} r\right)[h(s, u)-\ln (1+h(s, u))] \lambda(\mathrm{d} u) \mathrm{d} s .
\end{aligned}
$$

Substituting this inequality into (4.2) yields

$$
\begin{aligned}
\ln x(t) \leq & \exp \left(-\int_{0}^{t} a(s) \mathrm{d} s\right) \ln x_{0} \\
& +\int_{0}^{t} \exp \left(-\int_{s}^{t} a(r) \mathrm{d} r\right)\left(a(s) b(s)-1-\frac{1}{2} \sigma^{2}(s)\right) \mathrm{d} s \\
& +\frac{\epsilon \exp \left(-\int_{n \gamma}^{t} a(s) \mathrm{d} s\right)}{2} \int_{0}^{t} \exp \left(\int_{0}^{s} 2 a(r) \mathrm{d} r\right) \sigma^{2}(s) \mathrm{d} s \\
& +\int_{0}^{t} \int_{\mathbb{Y}} \exp \left(-\int_{s}^{t} a(r) \mathrm{d} r\right)[h(s, u)-\ln (1+h(s, u))] \lambda(\mathrm{d} u) \mathrm{d} s \\
& +\frac{\theta \exp \left(-\int_{n \gamma}^{t} a(s) \mathrm{d} s\right) \ln n}{\epsilon} .
\end{aligned}
$$


Then, for any $\omega \in \Omega_{i}$ and $(n-1) \gamma \leq t \leq n \gamma$ with $n \geq n_{0}+1$, we get

$$
\begin{aligned}
\frac{\ln x(t)}{\ln t} \leq & \exp \left(-\int_{0}^{t} a(s) \mathrm{d} s\right) \frac{\ln x_{0}}{\ln t}+\frac{1}{\ln t} \int_{0}^{t} \exp \left(-\int_{s}^{t} a(r) \mathrm{d} r\right) \\
& \times\left(a(s) b(s)-1-\frac{1}{2} \sigma^{2}(s)\right) \mathrm{d} s+\frac{\epsilon \exp \left(-\int_{n \gamma}^{t} a(s) \mathrm{d} s\right)}{2 \ln t} \\
& \times \int_{0}^{t} \exp \left(\int_{0}^{s} 2 a(r) \mathrm{d} r\right) \sigma^{2}(s) \mathrm{d} s+\frac{\theta \exp \left(-\int_{n \gamma}^{t} a(s) \mathrm{d} s\right) \ln n}{\epsilon \ln t} \\
& +\frac{1}{\ln t} \int_{0}^{t} \int_{\mathbb{Y}} \exp \left(-\int_{s}^{t} a(r) \mathrm{d} r\right)[h(s, u)-\ln (1+h(s, u))] \lambda(\mathrm{d} u) \mathrm{d} s \\
\leq & \exp \left(-\int_{0}^{t} a(s) \mathrm{d} s\right) \frac{\ln x_{0}}{\ln t}+\frac{1}{\ln t} \int_{0}^{t} \exp \left(-\int_{s}^{t} a(r) \mathrm{d} r\right) \\
& \times\left(a(s) b(s)-\frac{1}{2}\left(1-\epsilon \exp \left(\int_{n \gamma}^{s} a(r) \mathrm{d} r\right) \sigma^{2}(s)\right) \mathrm{d} s\right. \\
& +\frac{\theta \exp \left(\int_{(n-1) \gamma}^{n \gamma} a(s) \mathrm{d} s\right) \ln n}{\epsilon \ln (n-1) \gamma}+\frac{1}{\ln t} \\
& \times \int_{0}^{t} \int_{\mathbb{Y}} \exp \left(-\int_{s}^{t} a(r) \mathrm{d} r\right)[h(s, u)-\ln (1+h(s, u))] \lambda(\mathrm{d} u) \mathrm{d} s .
\end{aligned}
$$

Taking $n \uparrow \infty$, together with (4.1), leads to

$$
\limsup _{t \rightarrow \infty} \frac{\ln x(t)}{\ln t} \leq \frac{\theta \mathrm{e}^{\check{a} \gamma}}{\epsilon}
$$

Setting $\gamma \downarrow 0, \epsilon \uparrow 1$, and $\theta \uparrow 1$ leads to

$$
\limsup _{t \rightarrow \infty} \frac{\ln x(t)}{\ln t} \leq 1
$$

Using the limit $\lim _{t \rightarrow \infty} \frac{\ln t}{t}=0$, we obtain that $\limsup _{t \rightarrow \infty} \frac{\ln x(t)}{t} \leq 0$.

Theorem 4.2 Let the conditions of Theorem 2.1 and Assumption A.3 hold. Assume further that

$$
\liminf _{t \rightarrow \infty} \frac{1}{t} \int_{0}^{t} a(s)(1+\ln b(s))-\frac{\sigma^{2}(s)}{2}-\int_{\mathbb{Y}}(h(s, u)-\ln (1+h(s, u))) \lambda(\mathrm{d} u) \mathrm{d} s>0
$$

Then the population $(x(t))$ is persistent in mean, that is,

$$
\liminf _{t \rightarrow \infty} \frac{1}{t} \int_{0}^{t} x(s) \mathrm{d} s>0 \quad \text { a.s. }
$$

Proof Applying Itô's formula, we obtain

$$
\begin{aligned}
\ln x(t)+\int_{0}^{t} a(s) \ln x(s) \mathrm{d} s= & \ln x_{0}+\int_{0}^{t} c(s) \mathrm{d} s+\int_{0}^{t} \sigma(s) \mathrm{d} B(s) \\
& +\int_{0}^{t} \int_{\mathbb{Y}} \ln (1+h(s, u)) \tilde{N}(\mathrm{~d} s, \mathrm{~d} u) .
\end{aligned}
$$


Using the inequality $\ln x \leq x-1$ for $x>0$ yields that

$$
\begin{aligned}
\ln x(t)+\int_{0}^{t} a(s) \ln x(s) \mathrm{d} s & \leq x(t)-1+\int_{0}^{t} a(s)(x(s)-1) \mathrm{d} s \\
& \leq \mathrm{e}^{-\check{a} t} \operatorname{de}^{\check{a} t} \int_{0}^{t} x(s) \mathrm{d} s-\int_{0}^{t} a(s) \mathrm{d} s-1 .
\end{aligned}
$$

Combining (4.3) and (4.4) leads to

$$
\begin{aligned}
\mathrm{e}^{-\breve{a} t} \mathrm{de}^{\check{a} t} \int_{0}^{t} x(s) \mathrm{d} s \geq & \ln x_{0}+\int_{0}^{t} c(s) \mathrm{d} s+\int_{0}^{t} \sigma(s) \mathrm{d} B(s) \\
& +\int_{0}^{t} \int_{\mathbb{Y}} \ln (1+h(s, u)) \tilde{N}(\mathrm{~d} s, \mathrm{~d} u)+\int_{0}^{t} a(s) \mathrm{d} s+1 .
\end{aligned}
$$

Multiplying both sides of the last inequality by $\mathrm{e}^{\check{a} t}$ yields that

$$
\begin{aligned}
\mathrm{de}^{\check{a} t} \int_{0}^{t} x(s) \mathrm{d} s \geq & \mathrm{e}^{\check{a} t} \ln x_{0}+\mathrm{e}^{\check{a} t} \int_{0}^{t} c(s) \mathrm{d} s+\mathrm{e}^{\check{a} t} \int_{0}^{t} \sigma(s) \mathrm{d} B(s) \\
& +\mathrm{e}^{\check{a} t} \int_{0}^{t} \int_{\mathbb{Y}} \ln (1+h(s, u)) \tilde{N}(\mathrm{~d} s, \mathrm{~d} u)+\mathrm{e}^{\check{a} t} \int_{0}^{t} a(s) \mathrm{d} s+\mathrm{e}^{\check{a} t} .
\end{aligned}
$$

Integrating both sides of the inequality from 0 to $t$, we deduce

$$
\begin{aligned}
\mathrm{e}^{\check{a} t} \int_{0}^{t} x(s) \mathrm{d} s \geq & \ln x_{0} \int_{0}^{t} \mathrm{e}^{\check{a} s} \mathrm{~d} s+\int_{0}^{t} \mathrm{e}^{\check{a} s} \mathrm{~d} s \int_{0}^{s} c(r) \mathrm{d} r+\int_{0}^{t} \mathrm{e}^{\check{a} s} \mathrm{~d} s \\
& \times \int_{0}^{s} \sigma(r) \mathrm{d} B(r)+\int_{0}^{t} \mathrm{e}^{\check{a} s} \mathrm{~d} s \int_{0}^{s} \int_{\mathbb{Y}} \ln (1+h(r, u)) \tilde{N}(\mathrm{~d} r, \mathrm{~d} u) \\
& +\int_{0}^{t} \mathrm{e}^{\check{a} s} \mathrm{~d} s \int_{0}^{s} a(r) \mathrm{d} r+\int_{0}^{t} \mathrm{e}^{\check{a} s} \mathrm{~d} s .
\end{aligned}
$$

This further gives that

$$
\begin{aligned}
\int_{0}^{t} x(s) \mathrm{d} s \geq & \frac{\left(1+\ln x_{0}\right)}{\check{a}}\left(1-\mathrm{e}^{-\check{a} t}\right)+\frac{1}{\check{a}} \int_{0}^{t} c(s) \mathrm{d} s-\frac{1}{\check{a}} \int_{0}^{t} \mathrm{e}^{\check{a}(s-t)} c(s) \mathrm{d} s \\
& +\frac{1}{\check{a}} \int_{0}^{t} \sigma(s) \mathrm{d} B(s)-\frac{1}{\check{a}} \int_{0}^{t} \mathrm{e}^{\check{a}(s-t)} \sigma(s) \mathrm{d} B(s) \\
& +\frac{1}{\check{a}} \int_{0}^{t} \int_{\mathbb{Y}} \ln (1+h(s, u)) \tilde{N}(\mathrm{~d} s, \mathrm{~d} u) \\
& -\frac{1}{\check{a}} \int_{0}^{t} \int_{\mathbb{Y}} \mathrm{e}^{\check{a}(s-t)} \ln (1+h(s, u)) \tilde{N}(\mathrm{~d} s, \mathrm{~d} u) \\
& +\frac{1}{\check{a}} \int_{0}^{t} a(s) \mathrm{d} s-\frac{1}{\check{a}} \int_{0}^{t} \mathrm{e}^{\check{a}(s-t)} a(s) \mathrm{d} s .
\end{aligned}
$$

On the other hand, let

$$
M_{2}(t)=\int_{0}^{t} \sigma(s) \mathrm{d} B(s), \quad M_{3}(t)=\int_{0}^{t} \int_{\mathbb{Y}} \ln (1+h(s, u)) \tilde{N}(\mathrm{~d} s, \mathrm{~d} u)
$$




$$
M_{4}(t)=\int_{0}^{t} \int_{\mathbb{Y}} \mathrm{e}^{\check{a}(s-t)} \sigma(s) \mathrm{d} B(s), \quad M_{5}(t)=\int_{0}^{t} \int_{\mathbb{Y}} \mathrm{e}^{\check{a}(s-t)} \ln (1+h(s, u)) \tilde{N}(\mathrm{~d} s, \mathrm{~d} u) .
$$

Compute by the boundedness of $\sigma$ that

$$
\begin{aligned}
& \left\langle M_{2}\right\rangle(t)=\left[M_{2}\right](t)=\int_{0}^{t} \sigma^{2}(s) \mathrm{d} s \leq \check{\sigma}^{2} t, \\
& \left\langle M_{4}\right\rangle(t)=\left[M_{4}\right](t)=\int_{0}^{t} \int_{\mathbb{Y}} \mathrm{e}^{2 \check{a}(s-t)} \sigma^{2}(s) \mathrm{d} s \leq \check{\sigma}^{2} t,
\end{aligned}
$$

where $\langle M\rangle(t):=\langle M, M\rangle(t)$ is Meyer's angle bracket process.

Moreover, by Assumption A.3 we have

$$
\left\langle M_{3}\right\rangle(t)=\int_{0}^{t} \int_{\mathbb{Y}}(\ln (1+h(s, u)))^{2} \lambda(\mathrm{d} u) \mathrm{d} s \leq L t
$$

and

$$
\left\langle M_{5}\right\rangle(t)=\int_{0}^{t} \int_{\mathbb{Y}} \mathrm{e}^{2 \check{a}(s-t)}(\ln (1+h(s, u)))^{2} \lambda(\mathrm{d} u) \mathrm{d} s \leq L t .
$$

Using a strong law of large numbers for local martingales (see [19]), we get

$$
\lim _{t \rightarrow \infty} \frac{M_{i}(t)}{t}=0 \quad \text { a.s., } i=2,3,4,5
$$

From this we see that

$$
\begin{aligned}
\liminf _{t \rightarrow \infty} \frac{\int_{0}^{t} x(s) \mathrm{d} s}{t} \geq & \liminf _{t \rightarrow \infty} \frac{\left(1+\ln x_{0}\right)}{t \check{a}}\left(1-\mathrm{e}^{-\check{a} t}\right)+\liminf _{t \rightarrow \infty} \frac{1}{\check{a} t} \int_{0}^{t} c(s) \mathrm{d} s \\
& -\liminf _{t \rightarrow \infty} \frac{1}{\check{a} t} \int_{0}^{t} \mathrm{e}^{\check{a}(s-t)} c(s) \mathrm{d} s+\liminf _{t \rightarrow \infty} \frac{M_{1}(t)}{\check{a} t} \\
& -\liminf _{t \rightarrow \infty} \frac{M_{3}(t)}{\check{a} t}+\liminf _{t \rightarrow \infty} \frac{M_{2}(t)}{\check{a} t}-\liminf _{t \rightarrow \infty} \frac{M_{4}(t)}{\check{a} t} \\
& +\liminf _{t \rightarrow \infty} \frac{1}{\check{a} t} \int_{0}^{t} a(s) \mathrm{d} s-\liminf _{t \rightarrow \infty} \frac{1}{\check{a} t} \int_{0}^{t} \mathrm{e}^{\check{a}(s-t)} a(s) \mathrm{d} s \\
= & \liminf _{t \rightarrow \infty} \frac{1}{\check{a} t} \int_{0}^{t} c(s) \mathrm{d} s-\liminf _{t \rightarrow \infty} \frac{1}{\check{a} t} \int_{0}^{t} \mathrm{e}^{\check{a}(s-t)} c(s) \mathrm{d} s \\
& +\liminf _{t \rightarrow \infty} \frac{1}{\check{a} t} \int_{0}^{t} a(s) \mathrm{d} s-\liminf _{t \rightarrow \infty} \frac{1}{\check{a} t} \int_{0}^{t} \mathrm{e}^{\check{a}(s-t)} a(s) \mathrm{d} s .
\end{aligned}
$$

Moreover, by Assumption A.2 we have

$$
\lim _{t \rightarrow \infty} \frac{1}{t}\left|\int_{0}^{t} \mathrm{e}^{\check{a}(s-t)} c(s) \mathrm{d} s\right| \leq \lim _{t \rightarrow \infty} \frac{1}{\check{a} t} C\left(1-\mathrm{e}^{-\check{a} t}\right)=0
$$

and, together with

$$
\liminf _{t \rightarrow \infty} \frac{1}{\check{a} t} \int_{0}^{t} \mathrm{e}^{\check{a}(s-t)} a(s) \mathrm{d} s \leq \lim _{t \rightarrow \infty} \frac{1}{t}\left(1-\mathrm{e}^{-\check{a} t}\right)=0,
$$


we then obtain that

$$
\begin{aligned}
\liminf _{t \rightarrow \infty} \frac{\int_{0}^{t} x(s) \mathrm{d} s}{t} \geq & \liminf _{t \rightarrow \infty} \frac{1}{t} \int_{0}^{t} a(s)(1+\ln b(s))-\frac{\sigma^{2}(s)}{2} \\
& -\int_{\mathbb{Y}}(h(s, u)-\ln (1+h(s, u))) \lambda(\mathrm{d} u) \mathrm{d} s>0 \quad \text { a.s. }
\end{aligned}
$$

Theorem 4.3 Let the conditions of Theorem 2.1 and Assumption A.3 hold. Assume further that

$$
\limsup _{t \rightarrow \infty} \frac{1}{t} \int_{0}^{t} c(s) \mathrm{d} s<0 .
$$

Then the population $(x(t))$ goes to extinction, that is,

$$
\lim _{t \rightarrow \infty} x(t)=0 \quad \text { a.s. }
$$

Proof According to (3.2) in the proof of Theorem 3.1, we have

$$
\begin{aligned}
x(t)= & \exp \left\{\exp \left(-\int_{0}^{t} a(s) \mathrm{d} s\right) \ln x_{0}\right\} \exp \left\{t \left\{\frac{1}{t} \int_{0}^{t} \exp \left(-\int_{s}^{t} a(r) \mathrm{d} r\right) c(s) \mathrm{d} s\right.\right. \\
& +\frac{1}{t} \int_{0}^{t} \exp \left(-\int_{s}^{t} a(r) \mathrm{d} r\right) \sigma(s) \mathrm{d} B(s) \\
& \left.\left.+\frac{1}{t} \int_{0}^{t} \int_{\mathbb{Y}} \exp \left(-\int_{s}^{t} a(r) \mathrm{d} r\right) \ln (1+h(s, u)) \tilde{N}(\mathrm{~d} s, \mathrm{~d} u)\right\}\right\} .
\end{aligned}
$$

Let

$$
\bar{M}_{4}(t)=\int_{0}^{t} \int_{\mathbb{Y}} \exp \left(-\int_{s}^{t} a(r) \mathrm{d} r\right) \sigma(s) \mathrm{d} B(s)
$$

and

$$
\bar{M}_{5}(t)=\int_{0}^{t} \int_{\mathbb{Y}} \exp \left(-\int_{s}^{t} a(r) \mathrm{d} r\right) \ln (1+h(s, u)) \tilde{N}(\mathrm{~d} s, \mathrm{~d} u) .
$$

Using the same arguments as for (4.6), it is easy to see that we now have the following result:

$$
\lim _{t \rightarrow \infty} \frac{\bar{M}_{i}(t)}{t}=0 \quad \text { a.s., } i=4,5 .
$$

Furthermore, in view of condition (4.8), we conclude that $\lim _{t \rightarrow \infty} x(t)=0$ a.s. The proof is complete.

Remark 4.1 Theorems 4.2 and 4.3 show that the extinction and persistence of this model both depend on the values of $\lim _{t \rightarrow \infty} \frac{1}{t} \int_{0}^{t} c(s) \mathrm{d} s$ and $\lim _{t \rightarrow \infty} \frac{1}{t} \int_{0}^{t} a(s) \mathrm{d} s$. If $\liminf _{t \rightarrow \infty} \frac{1}{t} \times$ $\int_{0}^{t}(a(s)+c(s)) \mathrm{d} s>0$, then the population $(x(t))$ is persistent in mean; If $\limsup _{t \rightarrow \infty} \frac{1}{t} \times$ $\int_{0}^{t} c(s) \mathrm{d} s<0$, then the population $(x(t))$ goes to extinction. Therefore, $\lim _{t \rightarrow \infty} \frac{1}{t} \int_{0}^{t} c(s) \mathrm{d} s$ is the threshold between the extinction and persistence in mean. 
Remark 4.2 For $c(t)=a(t) \ln b(t)-\frac{1}{2} \sigma^{2}(t)-\int_{\mathbb{Y}}[h(t, u)-\ln (1+h(t, u))] \lambda(\mathrm{d} u)$, if $\int_{\mathbb{Y}}[h(t, u)-$ $\ln (1+h(t, u))] \lambda(\mathrm{d} u) \geq a(t) \ln b(t)$, then (4.8) holds. By Theorem 4.3 we can find that a Lévy noise can suppress a potential explosion and make the population extinct. This is reasonable since, as large and sudden environmental disturbance happens, the population has little time to adapt to the sudden changes, which leads to an increase in mortality.

Remark 4.3 There are many papers on the persistence in mean and extinction for stochastic models, and a common means to do this is by using the stochastic comparison theorem and the sample Lyapunov exponent; see [7, 20]. In this paper, we use a new method to study the persistence in mean without using stochastic comparison theorem and Lyapunov exponent.

Remark 4.4 Assumption A.3 imposed on the jump-diffusion coefficient plays a vital role in the process of proof, but it is not unique. Here, we provide another assumption.

Assumption A.4 There are constants $\delta>-1$ and $C_{1}>0$ such that, for any $t \geq 0$,

$$
h(t, u) \geq \delta, \quad u \in \mathbb{Y},
$$

and

$$
\int_{\mathbb{Y}}(h(t, u))^{2} \lambda(\mathrm{d} u) \leq C_{1}
$$

This assumption and the fact $\ln x \leq x-1(x>0)$ give, for $-1<\delta \leq 0$ and any $t \geq 0$,

$$
\begin{aligned}
|\ln (1+h(t, u))| & \leq\left|\ln (1+h(t, u)) I_{\delta \leq h(t, u) \leq 0}\right|+\left|\ln (1+h(t, u)) I_{h(t, u) \geq 0}\right| \\
& \leq-\ln (1+\delta)+|h(t, u)| .
\end{aligned}
$$

It now follows that

$$
\begin{aligned}
\int_{\mathbb{Y}}(\ln (1+h(t, u)))^{2} \lambda(\mathrm{d} u) & \leq 2(-\ln (1+\delta))^{2} \lambda(\mathbb{Y})+2 \int_{\mathbb{Y}} h^{2}(t, u) \lambda(\mathrm{d} u) \\
& \leq 2(-\ln (1+\delta))^{2} \lambda(\mathbb{Y})+2 C_{1}=: \bar{C}
\end{aligned}
$$

Consequently, using Assumption A.4 in lieu of Assumption A.3 yields that the results of Theorem 4.2 and 4.3 are still tenable.

Theorem 4.4 Under the conditions of Theorem 4.1, assume further that

$$
\limsup _{t \rightarrow \infty} \frac{1}{t} \int_{0}^{t} a(s)(1+\ln b(s))-\frac{\sigma^{2}(s)}{2}-\int_{\mathbb{Y}}(h(s, u)-\ln (1+h(s, u))) \lambda(\mathrm{d} u) \mathrm{d} s>0 .
$$

Then the population $(x(t))$ is weakly persistent, that is,

$$
\limsup _{t \rightarrow \infty} x(t)>0 \quad \text { a.s. }
$$


Proof If $P\left(\lim _{t \rightarrow \infty} x(t)=0\right)>0$, there exists a measurable subset $\Omega^{\prime}$ of $\Omega$ such that $P\left(\Omega^{\prime}\right)>$ 0 and $\lim _{t \rightarrow \infty} x(t, \omega)=0$ for any $\omega \in \Omega^{\prime}$. Then by Itô's formula,

$$
\begin{aligned}
\ln x(t)= & \ln x_{0}+\int_{0}^{t}[c(s)-a(s) \ln x(s)] \mathrm{d} s+\int_{0}^{t} \sigma(s) \mathrm{d} B(s) \\
& +\int_{0}^{t} \int_{\mathbb{Y}} \ln (1+h(s, u)) \tilde{N}(\mathrm{~d} s, \mathrm{~d} u) \\
\geq & \ln x_{0}+\int_{0}^{t}[c(s)+a(s)-a(s) x(s)] \mathrm{d} s+\int_{0}^{t} \sigma(s) \mathrm{d} B(s) \\
& +\int_{0}^{t} \int_{\mathbb{Y}} \ln (1+h(s, u)) \tilde{N}(\mathrm{~d} s, \mathrm{~d} u) .
\end{aligned}
$$

Combining this with (4.6), for all $\omega \in \Omega^{\prime}$, we then obtain

$$
\begin{aligned}
\limsup _{t \rightarrow \infty} \frac{\ln x(t)}{t}= & \limsup _{t \rightarrow \infty} \frac{1}{t} \int_{0}^{t}\left[a(s)(1+\ln b(s))-\frac{\sigma^{2}(s)}{2}\right. \\
& \left.-\int_{\mathbb{Y}}[h(s, u)-\ln (1+h(s, u))] \lambda(\mathrm{d} u)\right] \mathrm{d} s>0 .
\end{aligned}
$$

This contradicts with the fact that $\limsup _{t \rightarrow \infty} \frac{\ln x(t)}{t} \leq 0$ in Theorem 4.1. So $(x(t))$ is weakly persistent.

\section{Numerical simulations}

In this section, in order to testify the validity of the main results, we introduce the following examples and simulations.

Example 5.1 Let $x_{0}=2.0, a(t)=0.2, b(t)=\mathrm{e}, \sigma(t)=0.03, \lambda(\mathbb{Y})=1$, and the step size $\Delta t=0.01$. The only difference between of Figure 1 and Figure 2 is that the value of jumpdiffusion $h$ are different. In Figure 1 , we choose $h(t, u)=0.2406$. Then by a simple calculation we have

$$
\int_{\mathbb{Y}}\left[h_{1}(u, t)-\ln \left(1+h_{1}(u, t)\right)\right] \lambda(\mathrm{d} u)=0.025
$$

and

$$
a(t)(1+\ln b(t))-\frac{\sigma^{2}(t)}{2}-\int_{\mathbb{Y}}\left[h_{1}(u, t)-\ln \left(1+h_{1}(u, t)\right)\right] \lambda(\mathrm{d} u)=0.3732>0 .
$$

Figure 1 Solutions of system (1.1) for $x_{0}=2.0$, $a(t)=0.2, b(t)=\mathrm{e}, \sigma(t)=0.03, h(t, u)=0.2406$, $\lambda(\mathbb{Y})=1$, and the step size $\Delta t=0.01$.

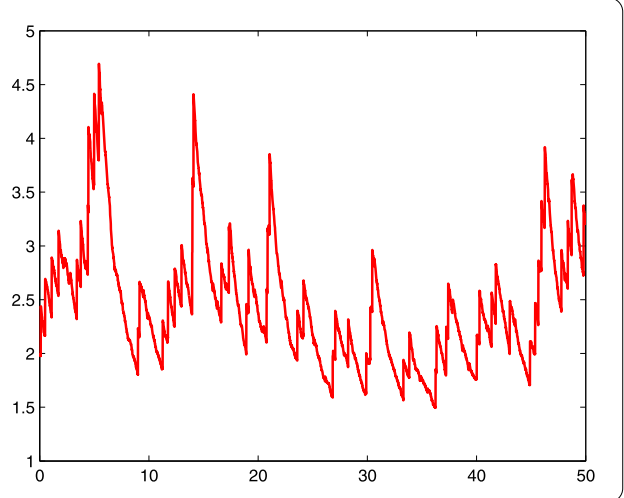




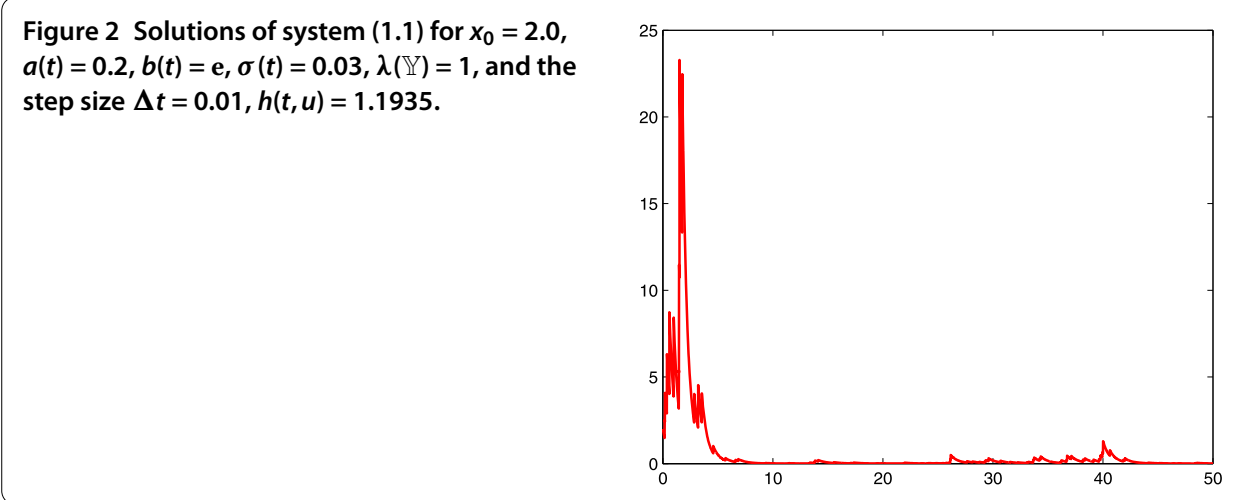

Figure 3 Solutions of system (1.1) for $x_{0}=2.0$, $a(t)=0.3, b(t)=\mathrm{e}, \sigma(t)=0.04, \lambda(\mathbb{Y})=1$, and the step size $\Delta t=0.01, h(t, u)=0.4863$.

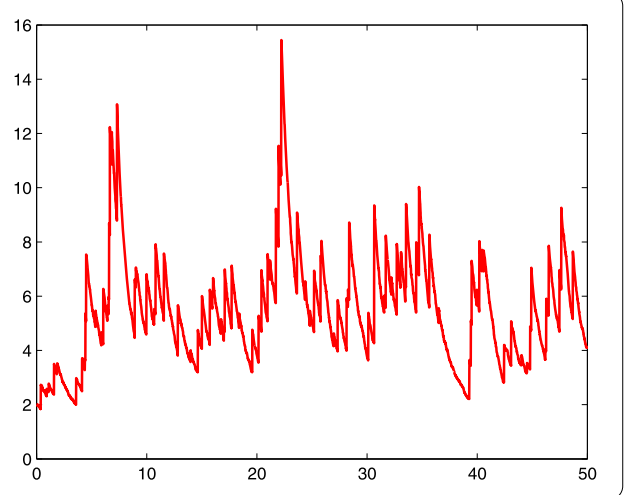

Then by Theorem 4.2, $(x(t))$ is persistent in mean, and Figure 1 confirms this. In Figure 2, we choose $h(t, u)=1.1935$, and then by a simple calculation we have

$$
\int_{\mathbb{Y}}[h(u, t)-\ln (1+h(u, t))] \lambda(\mathrm{d} u)=0.408
$$

and

$$
c(t)=a(t) \ln b(t)-\frac{\sigma^{2}(t)}{2}-\int_{\mathbb{Y}}[h(u, t)-\ln (1+h(u, t))] \lambda(\mathrm{d} u)=-0.2098<0 .
$$

In view of Theorem 4.3, we see that $(x(t))$ goes to extinction, and Figure 2 confirms this.

Example 5.2 Let $x_{0}=2.0, a(t)=0.3, b(t)=\mathrm{e}, \sigma(t)=0.04, \lambda(\mathbb{Y})=1$, and the step size $\Delta t=$ 0.01 . In Figure 3 , we choose $h(t, u)=0.4863$, and then by a simple calculation we have

$$
\int_{\mathbb{Y}}\left[h_{1}(u, t)-\ln \left(1+h_{1}(u, t)\right)\right] \lambda(\mathrm{d} u)=0.09
$$

and

$$
a(t)(1+\ln b(t))-\frac{\sigma^{2}(t)}{2}-\int_{\mathbb{Y}}\left[h_{1}(u, t)-\ln \left(1+h_{1}(u, t)\right)\right] \lambda(\mathrm{d} u)=0.8092>0
$$




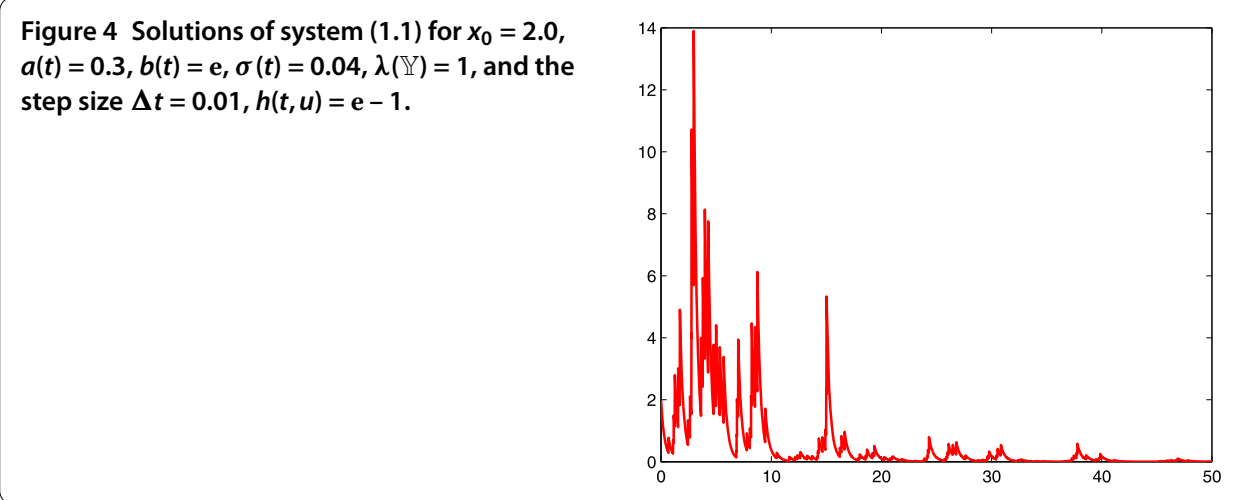

Then, by Theorem 4.2, $(x(t))$ is persistent in mean, and Figure 3 confirms this. In Figure 4, we choose $h(t, u)=\mathrm{e}-1$, and then by a simple calculation we have

$$
\int_{\mathbb{Y}}[h(u, t)-\ln (1+h(u, t))] \lambda(\mathrm{d} u)=\mathrm{e}-2
$$

and

$$
c(t)=a(t) \ln b(t)-\frac{\sigma^{2}(t)}{2}-\int_{\mathbb{Y}}[h(u, t)-\ln (1+h(u, t))] \lambda(\mathrm{d} u)=-0.1191<0 .
$$

According to Theorem 4.3, we obtain that $(x(t))$ goes to extinction.

The only difference between conditions of Figure 1 and Figure 2 (or Figure 3 and Figure 4) in these examples is that the values of jump-diffusion are different. This confirms that a Lévy noise plays an important role in population dynamics.

\section{Conclusion}

In this work, we propose a stochastic nonautonomous Gompertz model driven by Lévy jumps. We show that the model admits a unique global positive solution and study asymptotic moment properties of solutions. Moreover, we provide sufficient conditions for the extinction, persistence in the mean, and weak persistence of this model. The results confirm that the intensity of jump noise has a grave impact on the properties of this model. In the future, we will study some more practical and complex models such as the hybrid system driven by continuous-time Markov chains.

\section{Competing interests}

The authors declare that they have no competing interests.

Authors' contributions

All authors contributed equally to the manuscript. All authors read and approved the final manuscript.

Author details

${ }^{1}$ School of Mathematics and Statistics, Central South University, Changsha, 410083, China. ${ }^{2}$ College of Electrical and Information Engineering, Hunan University of Technology, Zhuzhou, 412007, China. 
References

1. $\mathrm{Hu}, \mathrm{G}$ : Invariant distribution of stochastic Gompertz equation under regime switching. Math. Comput. Simul. 97, 192-206 (2014)

2. Ferrante, L, Bompadre, S, Possati, L, Leone, L: Parameter estimation in a Gompertzian stochastic model for tumor growth. Biometrics 56, 1076-1081 (2000)

3. Ferrante, L, Bompadre, S, Leone, L, Montanari, MP: A stochastic formulation of the Gompertzian growth model for in vitro bactericidal kinetics: parameter estimation and extinction probability. Biom. J. 47, 309-318 (2005)

4. Jovanovic, M, Krstic, M: Analysis of non-autonomous stochastic Gompertz model with delay. Appl. Math. Comput. 242, 101-108 (2014)

5. Lo, CF: Stochastic Gompertz model of tumour cell growth. J. Theor. Biol. 248, 317-321 (2007)

6. Moummou, EK, Gutierrez-Sanchez, R, Melchor, MC: A stochastic Gompertz model highlighting internal and external therapy function for tumour growth. Appl. Math. Comput. 246, 1-11 (2014)

7. Bao, J, Yuan, C: Stochastic population dynamics driven by Lévy noise. J. Math. Anal. Appl. 391, $363-375$ (2012)

8. Bao, J, Yuan, C: Numerical analysis for neutral SPDEs driven by $\alpha$-stable processes. Infin. Dimens. Anal. Quantum Probab. Relat. Top. (2014). doi:10.1142/S0219025714500313

9. Bao, J, Mao, X, Yin, G, Yuan, C: Competitive Lotka-Volterra population dynamics with jumps. Nonlinear Anal. 74, 6601-6616 (2011)

10. Bao, J, Yuan, C: Long-term behavior of stochastic interest rate models with jumps and memory. Insur. Math. Econ. 53, 266-272 (2013)

11. Bao, J, Yuan, C: Large deviations for neutral SDEs with jumps. Stochastics 87, 48-70 (2015)

12. Bao, J, Yuan, C: Blow-up for stochastic reaction-diffusion equations with jumps. J. Theor. Probab. 29(2), 617-631 (2016)

13. Bao, J, Truman, A, Yuan, C: Stability in distribution of mild solutions to stochastic partial differential delay equations with jumps. Proc. R. Soc. Lond., Ser. A, Math. Phys. Eng. Sci. 465, 2111-2134 (2009)

14. Hou, Z, Bao, J, Yuan, C: Exponential stability of energy solutions to stochastic partial differential equations with variable delays and jumps. J. Math. Anal. Appl. 366, 44-54 (2010)

15. Liu, Q: Asymptotic properties of a stochastic $n$-species Gilpin-Ayala competitive model with Lévy jumps and Markovian switching. Commun. Nonlinear Sci. Numer. Simul. 26, 1-10 (2015)

16. Liu, Q: Asymptotic behavior of a stochastic non-autonomous predator-prey system with jumps. Commun. Nonlinear Sci. Numer. Simul. 26, 1-10 (2015)

17. Peng, S, Zhu, X: Necessary and sufficient condition for comparison theorem of 1-dimensional stochastic differential equations. Stoch. Process. Appl. 116, 370-380 (2006)

18. Applebaum, D: Lévy Processes and Stochastics Calculus, 2nd edn. Cambridge University Press, Cambridge (2009)

19. Liptser, R: A strong law of large numbers for local martingales. Stochastics 3, 217-228 (1980)

20. Liu, Q, Chen, Q: Analysis of a general stochastic non-autonomous logistic model with delays and Lévy jumps. J. Math. Anal. Appl. 433, 95-120 (2016)

\section{Submit your manuscript to a SpringerOpen ${ }^{\circ}$ journal and benefit from:}

- Convenient online submission

Rigorous peer review

- Immediate publication on acceptance

- Open access: articles freely available online

- High visibility within the field

- Retaining the copyright to your article 